\title{
A Influência da Personalidade do Aluno na Construção de Grupos de Trabalho em Sala de Aula
}

\author{
André V. Tardelli ${ }^{1}$, Juliana B. dos S. França², Angélica F. S. Dias ${ }^{3}$, Adriana S. \\ Vivacqua $^{1,4}$, Marcos R. da S. Borges ${ }^{1,4}$ \\ ${ }^{1}$ Departamento de Ciência da Computação (DCC) - Universidade Federal do Rio de \\ Janeiro (UFRJ) - Rio de Janeiro, Brasil. \\ ${ }^{2}$ Departmentamento de Computação (DECOMP) - Universidade Federal Rural do Rio \\ de Janeiro (UFRRJ) - Rio de Janeiro, Brasil. \\ ${ }^{3}$ Núcleo de Computação Eletrônica (NCE) - Universidade Federal do Rio de Janeiro \\ (UFRJ) - Rio de Janeiro, Brasil. \\ ${ }^{4}$ Programa de Pós-Graduação em Informática - Universidade Federal do Rio de Janeiro \\ (UFRJ) - Rio de Janeiro, Brasil. \\ \{andretardelli, julibsf, angelicafsdias\}@gmail.com, \{avivacqua, \\ mborges\} @ppg • ufrj.br
}

\begin{abstract}
This article presents the first discussions on work groups definition in classroom, based on the influence of the student's personality in this organization. It is known that groups organized both technically and emotionally can result in solutions closer to desirable. Thus, through an analysis of its components by a psychological bias, it is possible to form groups that are more compatible with the needs of the task being performed. In order to optimize this categorization, a work group definition framework was proposed. Based on indicators such as the Myers-Briggs typology, it consists of a different combination of psychological profile according to the type of activity, stimulating the members evolution in a gradual way. This research had its pilot experiment conducted in a class of a computer science course in a Brazilian federal university, showing greater collaboration in groups of mixed profiles in different types of tasks
\end{abstract}

Resumo. Este artigo apresenta as primeiras discussões sobre a construção de grupos de trabalho em sala de aula, tendo por fundamento a influência da personalidade do aluno nesta organização. Entende-se que grupos organizados tanto tecnicamente, quanto emocionalmente podem resultar em soluções mais próximas do desejável. Assim, através de uma análise de seus componentes por um viés psicológico, é possível formar grupos que possuam maior compatibilidade com as necessidades da tarefa a ser realizada. De maneira a otimizar essa categorização, foi proposto o Framework para Formação de Grupos de Trabalho (FGT). Fundamentado em indicadores como a Tipologia de Myers-Briggs, este consiste em diferentes combinações de perfis psicológicos de acordo com o tipo de atividade, estimulando a evolução dos integrantes de forma gradual. Esta pesquisa teve seu experimento piloto conduzido no curso de ciência da computação em uma universidade federal 
VIII Congresso Brasileiro de Informática na Educação (CBIE 2019)

Anais do XXX Simpósio Brasileiro de Informática na Educação (SBIE 2019)

brasileira, mostrando maior interação colaborativa em grupos de perfis mistos em diferentes tipos de tarefas

\section{Introdução}

Atividade em grupo tem por objetivo promover o desempenho cognitivo, afetivo e social do aluno, além de colaborar com a construção de saberes através do compartilhamento das diferentes visões sobre um assunto [Gessinger 2008]. Segundo Silva et al. (2017) a sala de aula reúne as características propícias para a estabelecimento de tarefas em grupo.

As atividades em grupo em sala de aula tratam-se de uma excelente oportunidade de descontinuar a proposta de sala de aula expositiva e desenvolver iniciativas que favoreçam o posicionamento do aluno como protagonista em seu processo de aprendizagem. Os trabalhos em grupo realizados em sala de aula são uma oportunidade de promover a interação e cooperação entre os alunos [Gessinger 2008]. No entanto, para estas ocorrerem, não basta juntar alunos em grupos, é necessário estruturar a tarefa a ser desempenhada e organizar os objetivos a serem alcançados.

Sendo utilizados como pilares na base educacional, trabalhos em grupo estimulam a colaboração, delegam autoridade e o convívio social entre os alunos [Cohen e Lotan 2017]. Todavia, muitas vezes ocorrem situações onde determinadas configurações de grupo são incompatíveis na realização de tarefas, seja pela distinção dos processos de tomada de ações dos indivíduos ou mesmo no conflito de ideologias. Nestes casos, é notável a ocorrência de consequências, como a perda de motivação e objetivos comuns entre os participantes. Diante deste cenário, é proposto o estudo de diferentes configurações entre tipos de atividades em grupo, de forma a evitar possíveis conflitos e obter perfis de grupos mais compatíveis.

De acordo com as mais abrangentes teorias da psicologia, cada pessoa possui um perfil psicológico que define as suas características externas principais e seus padrões de processos de tomada de decisão. Assim, é possível discernir estes indivíduos em diferentes categorias, de modo a organizá-los quanto à suas preferências e talentos principais em uma atividade. Devido a isso, é possível formar grupos balanceados que complementam as fraquezas e forças dos componentes, de maneira que ainda seja possível prever possíveis compatibilidades entre esses grupos. Além disso, é possível propor tarefas configuradas de maneira a favorecer o aprendizado e manter o estado de motivação do aluno de tal forma que estimule a construção de saberes [Ribeiro et al. 2013].

Oliveira et al. (2018) discute através de uma revisão sistemática a identificação automática de estilos de aprendizagem em salas de aula, considerando a personalidade do aluno. No entanto, [Felder e Brent 2005] e [Farias et al. 2014] entendem que a adaptação dos métodos de aprendizagem para os diferentes tipos de alunos e suas personalidades continua sendo um desafio. Para tanto, é preciso alinhar os traços da personalidade do aluno e seu estado cognitivo com os processos de aprendizagem, que no caso desta pesquisa, visa-se explorar a aprendizagem colaborativa.

$\mathrm{Na}$ investigação discutida por este artigo, o objeto de estudo principal trata-se da formação de grupos na aprendizagem colaborativa. Espera-se com esta investigação levantar os principais indícios que favoreçam a construção de grupos de trabalho por 
VIII Congresso Brasileiro de Informática na Educação (CBIE 2019)

Anais do XXX Simpósio Brasileiro de Informática na Educação (SBIE 2019)

alunos com personalidades complementares, a fim de conduzir tarefas colaborativas em sala de aula física ou virtual.

Como solução proposta nesta pesquisa, tem-se uma fundamentação direta na teoria de Myers-Briggs Typology (MBTI) [Myers e Myers 1995]. Nela, é possível diagnosticar as características de cada indivíduo através de um conjunto alternado de dicotomias. De acordo com as mesmas, esta pesquisa propõe um conjunto de caracterizadores (em acrônimos) que auxiliam o professor ou facilitador em sala de aula na construção de grupos de trabalho que sejam compostos por alunos com personalidades complementares e aderentes as atividades em grupo. A construção dessas regras está fundamentada não apenas nas personalidades propostas e defendidas pelo modelo MBTI, mas também nas relações com as características da atividade em grupo e na visão e experiência dos autores.

Assim, este artigo visa apresentar as primeiras discussões sobre a construção de grupos de trabalho em ambientes educacionais, abordando um viés psicológico para seus componentes. Espera-se com isso traçar perfis de acordo com as necessidades de cada atividade e com o foco nos interesses do aluno. Para isso, são discutidos tipos de atividades conduzidas com alunos em sala de aula e também foi proposto o Framework para a formação de grupos de trabalho (FGT). Esta pesquisa teve seu experimento piloto conduzido no curso de ciência da computação em uma universidade federal brasileira.

O artigo é organizado em quatro seções, conforme apresentado a seguir. A seção 2 apresenta discussões a respeito de atividades em sala de aula, a Seção 3 discute a Tipologia de Myers-Briggs ou MBTI aplicado na formação de grupos de trabalho de aprendizagem e apresenta o Framework para a formação de grupos de trabalho (FGT). A Seção 4 apresenta breves resultados sobre um experimento piloto conduzido em curso de ciência da computação em uma universidade federal brasileira. A última seção apresenta as discussões finais sobre essa pesquisa e seus próximos passos.

\section{Atividades em Grupo e Em Sala de Aula}

Segundo Ciampone e Peduzzi (2000), “Um grupo é constituído por um conjunto de pessoas que se reúnem em um determinado espaço de tempo e lugar, tendo um objetivo em comum". No contexto educacional, trabalhos em grupo são utilizados como formas de estimular a colaboração e o convívio social entre os alunos. As atividades em grupo tratam-se de uma oportunidade de descontinuar a proposta de sala de aula expositiva e desenvolver iniciativas que favoreçam o aluno como protagonista em seu processo de aprendizagem.

Ao optar por trabalhos em grupo, o educador precisa ressaltar os motivos que o levaram a escolher tal opção. A interação e a cooperação entre os alunos não se dão de forma espontânea, então é preciso implementar atividades e designar tarefas que estimulem os alunos a colaborarem entre si [Gessinger 2008]. Caso os objetivos e os papéis de cada integrante não estejam bem definidos durante a realização do mesmo, é notável a falta de motivação para a conclusão de tarefas e a perda de objetivo comum entre os demais.

Desta maneira, estudos têm sido realizados para analisar as novas dinâmicas que promovem a motivação dentre todos os seus participantes [Niclhols e Miller, 1994]. Uma das tarefas para obter uma configuração de um grupo compatível envolve a garantia da satisfação de todas as necessidades socioemocionais de seus participantes, 
VIII Congresso Brasileiro de Informática na Educação (CBIE 2019)

Anais do XXX Simpósio Brasileiro de Informática na Educação (SBIE 2019)

de modo a destacar o papel de cada integrante na realização do objetivo em si [Toseland e Rivas 2005]. Assim, o conhecimento mais aprofundado do estudo destas dinâmicas é essencial para entender a estrutura social dos grupos no ambiente escolar.

\section{Formação de Grupos de Trabalho Considerando a Personalidade dos Alunos}

No ponto de vista cognitivo, pesquisas mostram que o entendimento das características de seus integrantes e o reconhecimento de seus papéis podem melhorar o desempenho de um grupo, desde que tal ciência seja utilizada para atribuir tarefas que otimizem os traços fortes desses integrantes. Analogamente, seguindo uma vertente emocional, estados afetivos e o conhecimento dos perfis de cada integrante podem contribuir também para a performance de um grupo, de modo a apaziguar situações de conflito e contribuir a um consenso entre alunos [Van Knippenberg e Schippers 2007]. Dessa forma, é possível notar que a diversidade de um grupo pode otimizar a carga organizacional e pessoal de trabalho em grupo, desde que implementados de forma correta em uma configuração compatível.

Tabela 1. Dicotomias das tipologias de Myers-Briggs, separadas por seus pares [3].

\begin{tabular}{l|c|c|c|c}
\hline \multicolumn{1}{c|}{ Caracterização } & \multicolumn{3}{c}{ Dicotomias } \\
\hline Sua fonte de energia. & E & Extroversão & I & Introversão \\
\hline Seu modo de perceber o mundo. & S & Sensação & N & Intuição \\
\hline Sua maneira de decidir e lidar com emoções. & T & Pensamento & F & Sentimento \\
\hline Sua abordagem no trabalho, planejamento e tomada de decisões. & J & Julgamento & P & Percepção \\
\hline
\end{tabular}

A Tipologia de Myers-Briggs ou MBTI categoriza estes indivíduos em diferentes dicotomias, de modo a organizá-los quanto à suas preferências e talentos principais em uma atividade. Devido a isso, é possível formar grupos equilibrados de acordo com a personalidade de seus componentes. A Tabela 1 apresenta as dicotomias defendidas por esta teoria.

\begin{tabular}{|c|c|c|c|}
\hline $\begin{array}{c}\text { ISTJ } \\
\text { Executores } \\
\text { responsáveis }\end{array}$ & $\begin{array}{c}\text { ISFJ } \\
\text { Administradores } \\
\text { dedicados }\end{array}$ & $\begin{array}{c}\text { INFJ } \\
\begin{array}{c}\text { Motivadores } \\
\text { Perpicazes }\end{array}\end{array}$ & $\begin{array}{c}\text { INTJ } \\
\text { Estrategistas } \\
\text { Visionarios }\end{array}$ \\
\hline $\begin{array}{c}\text { ISTP } \\
\text { Pragmáticos } \\
\text { Ageis }\end{array}$ & $\begin{array}{c}\text { ISFP } \\
\text { Guardiöes } \\
\text { Práticos }\end{array}$ & $\begin{array}{c}\text { INFP } \\
\text { Cruzados } \\
\text { Inspirados }\end{array}$ & $\begin{array}{c}\text { INTP } \\
\text { Analisadores } \\
\text { Expansivos }\end{array}$ \\
\hline $\begin{array}{c}\text { ESTP } \\
\text { Dinamistas } \\
\text { Dissidentes }\end{array}$ & $\begin{array}{c}\text { ESFP } \\
\text { Entusiastas } \\
\text { Improvisadores }\end{array}$ & $\begin{array}{c}\text { ENFP } \\
\text { Catalisadores } \\
\text { Veementes }\end{array}$ & $\begin{array}{c}\text { ENTP } \\
\text { Exploradores } \\
\text { Inovadores }\end{array}$ \\
\hline $\begin{array}{c}\text { ESTJ } \\
\text { Condutores } \\
\text { Eficientes }\end{array}$ & $\begin{array}{c}\text { ESFJ } \\
\text { Construtores } \\
\text { Comprometidos }\end{array}$ & $\begin{array}{c}\text { ENFJ } \\
\text { Mobilizadores } \\
\text { Envolventes }\end{array}$ & $\begin{array}{c}\text { ENTJ } \\
\text { Diretores } \\
\text { Estratégicos }\end{array}$ \\
\hline
\end{tabular}

Figura 1. Os 16 tipos de personalidade, definidos por seus acrônimos. 
Após a realização do teste, a personalidade do indivíduo é caracterizada em um acrônimo de quatro letras, representando quais dicotomias dos quatro pares o indivíduo mais se identifica (Figura 1). Desta forma, com a análise dos perfis psicológicos de cada integrante, é possível associá-las às atividades de trabalhos em grupo mais comuns, sinalizando de forma mais precisa ao educador de qual aluno poderia cumprir determinado papel de maneira mais eficiente em uma configuração. Atividades que demandam grande carga de trabalho escrito precisam de indivíduos que possuam maior talento na organização e designação de tarefas para cada integrante, enquanto atividades que fundamentam argumentações críticas necessitam de indivíduos que consigam balancear as opiniões individuais e estimular o consenso.

\section{Framework para a Formação de Grupos de Trabalho (FGT)}

Para construir um Framework de formação de grupos de trabalho (FGT), foram considerados os tipos de atividade em sala de aula [Gessinger 2008], construindo quatro categorias principais (Figura 2): (i) Pesquisa: Pesquisa e execução de relatórios escritos sobre determinado tema; (ii) Apresentação: Dissertações orais sobre determinado tema através de métodos como cartazes, slides, ppt, etc; (iii) Debate: Discussão entre um ou mais grupos envolvendo diferentes pontos de vista; e (iv) Criação: Elaboração de conteúdo, como maquetes, experimentos, conhecimentos, etc.

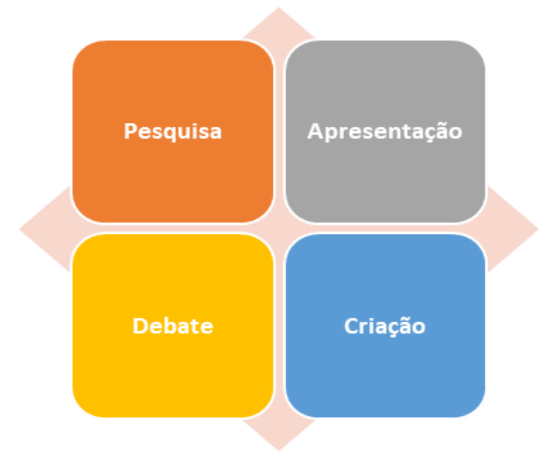

Figura 2. Categorias de atividades realizadas em sala de aula.

As categorias de atividades foram organizadas de acordo com as dicotomias (Tabela 1), formando dois grupos. O Grupo I passa a ser composto pelas dicotomias I/E que definem as atividades favoráveis para cada indivíduo. Já o Grupo II é composto por $\mathrm{N} / \mathrm{S}, \mathrm{F} / \mathrm{T}$ e J/P que definem quais traços de personalidade são pontos fortes do indivíduo para a atividade definida. Com base nesta organização, foi proposto o Framework para a formação de grupos de trabalho (FGT) (Figura 3).

Este Framework propõe que grupos de trabalho sejam estabelecidos em sala de aula, de acordo com a atividade fim. Ou seja, de acordo com uma das quatro possibilidades de atividades (pesquisa, apresentação, debate e criação), deve ser previsto um agrupamento das dicotomias que definem o traço de personalidade (expressado através de seus acrônimos) e sua relação com a introversão ou extroversão do indivíduo.

A Figura 3 defende que atividades de pesquisa requerem organização e planejamento (com dicotomias $\mathrm{S}$ e $\mathrm{J}$ ), pensamento $(\mathrm{T})$, julgamento $(\mathrm{J})$ e Observação $(\mathrm{S})$, apresentando uma tendência favorável para a atuação de alunos que já apresentam perfil introvertido, e para o estímulo daqueles alunos em competências compatíveis com a 
VIII Congresso Brasileiro de Informática na Educação (CBIE 2019)

Anais do XXX Simpósio Brasileiro de Informática na Educação (SBIE 2019)

característica de introversão. Com isso, atividades de pesquisa permeiam as dicotomias $\mathrm{T} / \mathrm{J} / \mathrm{S}$.

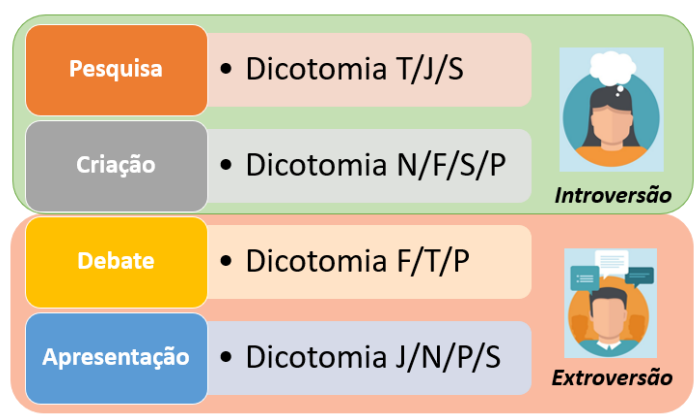

Figura 3. Framework FGT.

A atividade de criação exige características de criatividade (apresentando as dicotomias $\mathrm{S}$ e $\mathrm{P})$, observação $(\mathrm{S})$, percepção $(\mathrm{P})$, intuição $(\mathrm{N})$ e sentimento $(\mathrm{F})$, apresentando uma tendência favorável aos perfis introvertidos, assim como a tarefa de pesquisa. As atividades de Debate e Apresentação apresentam tendências extrovertidas. No entanto, as atividades de debate envolvem características como: consenso ( $\mathrm{F}$ e $\mathrm{P})$, percepção $(\mathrm{P})$, sentimento $(\mathrm{F})$, e pensamento $(\mathrm{T})$, gerando o acrônimo FTP. As atividades de Apresentação envolvem características como: Organização (S e J), criatividade ( $\mathrm{S}$ e $\mathrm{P}$ ), intuição $(\mathrm{N})$ e percepção $(\mathrm{P})$, gerando o acrônimo JNPS.

O Framework FGT foi proposto para auxiliar tanto o professor quanto o aluno em sala de aula, a fim de que ambos extraiam melhores resultados de suas experiências colaborativas na construção de seus saberes e na avaliação de desempenho. Para o professor, conhecer as características de personalidade de seus alunos favorece uma relação mais direta e maior proximidade, influenciando a forma de acompanhamento desse aluno e como ele deve ser cobrado ao longo de suas tarefas. Quanto aos alunos, estes são alocados em tarefas que gerem desconforto compatível com o grau que os alunos conseguem suportar e ao mesmo tempo evoluir seus saberes.

Todas as atividades em sala de aula discutidas por esta pesquisa (Figura 2) exigem interações colaborativas com seus pares em diferentes níveis de intensidade. Alunos com tendências introvertidas devem ser alocados não apenas nas atividades de Pesquisa e Criação, mas também em Debates e Apresentações a fim de que novas competências sejam formadas, considerando os avanços individuais de cada aluno. $\mathrm{O}$ mesmo deve ser considerado quanto à extroversão. Atividades como Pesquisa e Criação também devem ser desenvolvidas por alunos que não apresentam o perfil da introversão. Dessa maneira as características reflexivas e intuitivas são estimuladas.
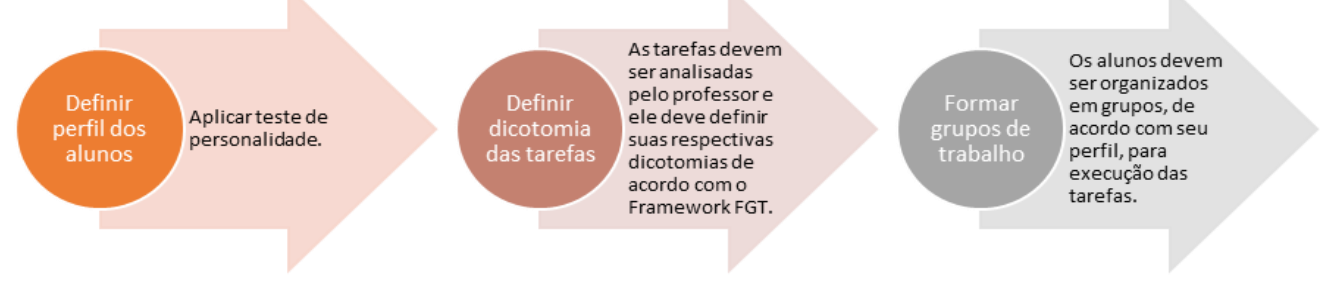

Figura 4. Processo Definir Grupos de Trabalho e seus subprocessos. 
VIII Congresso Brasileiro de Informática na Educação (CBIE 2019)

Anais do XXX Simpósio Brasileiro de Informática na Educação (SBIE 2019)

Para pôr em prática a aplicação do Framework FGT, deve-se pensar em um processo de formação dos grupos e suas respectivas ações associadas. A Figura 4 apresenta o conjunto de três subprocessos que compõem o processo Definir Grupos de Trabalho.

Para executar os subprocessos "Definir perfil do aluno" e "Definir dicotomia das tarefas" é necessário que os alunos realizem um teste de personalidade. Atualmente esta pesquisa usa o teste definido pelo 16Personalities ${ }^{1}$.

$\mathrm{Na}$ definição do perfil, busca-se descobrir se o aluno se identifica com os perfis Introvertido ou Extrovertido do Framework FGT. Com base nessas respostas, os alunos são categorizados de acordo com as dicotomias de cada tarefa a ser realizada por eles. Uma vez que os alunos estejam categorizados, os grupos são definidos de forma balanceada. Esse balanceamento leva em consideração a construção de saberes que desejam desenvolver com a atividade, considerando os interesses do professor e alunos.

\section{Experimentação da aplicação do Framework FGT}

A experimentação da construção de grupos de trabalhos com base no perfil psicológico dos alunos foi iniciada em uma turma de graduação do curso de ciência da computação de uma Universidade Federal Brasileira. O objetivo da experimentação era observar a influência do perfil dos alunos participantes nos resultados alcançados. Para este experimento, apenas uma atividade da Figura 2 foi investigada.

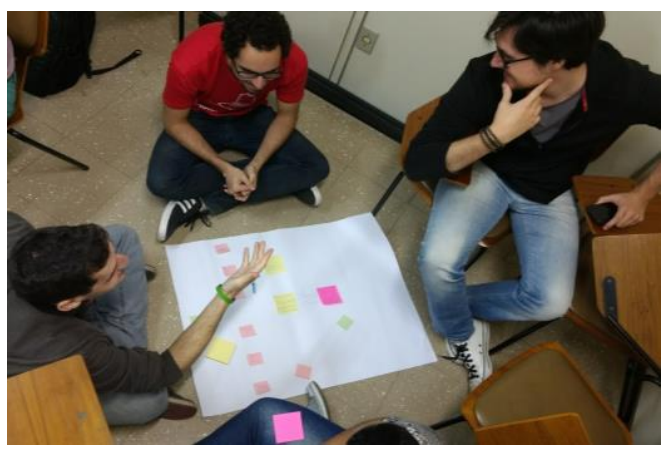

Figura 5: Grupo I - Atividade colaborativa de projeção de impactos.

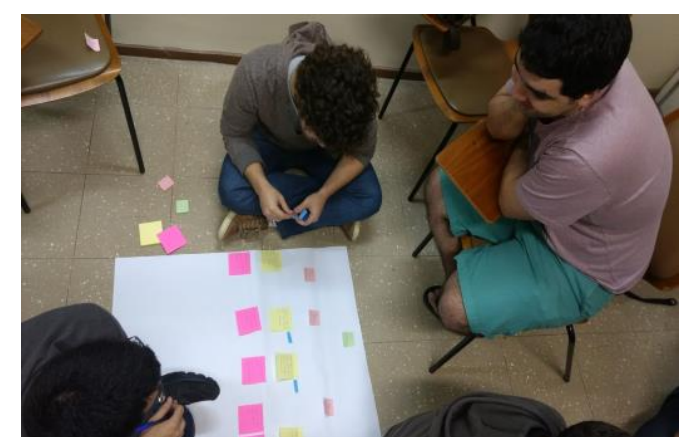

Figura 6: Grupo II - Atividade colaborativa de projeção de impactos.

A atividade proposta para os grupos em sala de aula foi de criação. Nela, os grupos precisaram projetar impactos, através de um modelo já conhecido, sobre uma situação de emergência no prédio em que eles assistem aulas (Figura 5 e Figura 6). Antes da atividade de criação colaborativa ser conduzida, os participantes responderam um teste de personalidade (16Personalities).

Durante o experimento, foi observado que alunos com personalidades mais racional e extrovertida (dicotomias $\mathrm{E}$ e $\mathrm{T}$ ), geraram resultados mais ricos para a construção do conhecimento coletivo. Em contrapartida, o excesso de componentes com este perfil acarretou conflitos internos no grupo, apresentando dificuldades na obtenção de um consenso. Já os grupos com componentes extrovertidos e introvertidos

\footnotetext{
${ }^{1}$ Free personality test. Acesso em Setembro/2018 em: https://www.16personalities.com/free-personality-
} test 
VIII Congresso Brasileiro de Informática na Educação (CBIE 2019)

Anais do XXX Simpósio Brasileiro de Informática na Educação (SBIE 2019)

(dicotomias E e I), apresentaram dificuldades na externalização do conhecimento e na construção do conhecimento coletivo.

Estes resultados mostram que a atividade de criação, que apresenta um viés de introversão, quando realizada em grupo, tende a estabelecer interações colaborativas que estimulam alunos com viés psicológico extrovertido a se integrarem às atividades com características mais introvertidas/reflexivas. Enquanto grupos que apresentaram componentes introvertidos, tiveram dificuldade em externar suas reflexões, intuição, percepção, sentimento e criatividade nas relações colaborativas que a atividade de criação proposta exigiu.

Os resultados mostram que a definição da atividade e das características pedagógicas, sociais e psicológicas são influenciadoras diretas na construção dos grupos de trabalho em sala de aula. Uma atividade como a de criação, quando configurada de forma a neutralizar a introversão e estimular a extroversão na incorporação de relações colaborativas, mostra que alunos inicialmente com o perfil compatível, apresentam dificuldade em se adaptar a este meio.

Outro aspecto observado é que grupos compostos por perfis mistos, ou seja, com participantes introvertidos e extrovertidos, tenderam a mostrar maior participação de alunos com perfil psicológico mais próximo da interação colaborativa. Como visto os alunos mais extrovertidos sobrepuseram os mais introvertidos e grupos com mais de um extrovertido dominante enfrentaram conflitos durante a resolução da tarefa. Apesar disso, os grupos com personalidades complementares apresentaram resultados mais discutidos e detalhados.

Por fim, este experimento mostrou que o teste de personalidade respondido no início do experimento refletiu resultados muito próximos do perfil do aluno participante. $\mathrm{O}$ experimento mostrou também que as dicotomias associadas às tarefas no Framework FGT estão de acordo com as características da atividade em sala de aula. Ainda como última constatação, esta investigação trouxe à luz que cada tarefa em sala de aula pode apresentar diferentes variações em sua formação e, portanto, estas precisam ser previstas a fim de que as dicotomias associadas sejam definidas.

\section{Conclusões e Próximos Passos}

Esta pesquisa visou discutir os primeiros passos para a construção de grupos em ambientes educacionais de acordo com a personalidade dos alunos. Ele abordou um viés psicológico para a construção de grupos de trabalho melhor estruturados, em função do desenvolvimento do aluno e seus saberes no processo de aprendizagem colaborativa. Neste artigo, observa-se que a construção de grupos em sala de aula vai mais além do que agrupar alunos e propor uma atividade. É preciso estruturar a tarefa, destacando os objetivos a serem alcançados para a construção de conhecimentos, e também diagnosticar perfis psicológicos que favoreçam o agrupamento de alunos de forma saudável para a busca dos resultados.

Neste artigo foi apresentado também o Framework FGT. Sua proposta é considerar não apenas o desenvolvimento de alunos já preparados tecnicamente e de acordo com suas personalidades para a participação nos grupos de trabalho, mas também na evolução de alunos com relação às suas aptidões menos desenvolvidas em função de sua personalidade. Este Framework foi investigado em um experimento 
VIII Congresso Brasileiro de Informática na Educação (CBIE 2019)

Anais do XXX Simpósio Brasileiro de Informática na Educação (SBIE 2019)

piloto, a fim de avaliar as dicotomias propostas para a atividade "Criação" prevista nele e também avaliar o comportamento dos perfis diante de uma atividade proposta.

Como próximos passos, esta pesquisa visa conduzir novos experimentos com a finalidade de investigar as demais tarefas previstas no Framework FGT. Além disso, é previsto propor um conjunto de heurísticas que sejam capazes de apoiar o professor /facilitador na construção de grupos de trabalho de acordo com as personalidades dos alunos e tipos de tarefas (detalhamento do subprocesso "Definir Grupos" do processo da Figura 4). Essas tarefas visam considerar as variações propostas para cada atividade, como foi o caso investigado no experimento com a atividade de criação com interações colaborativas entre os pares. Para isso, uma nova revisão da literatura está sendo conduzida a fim de que novos tipos de tarefa em grupo sejam levantados e relacionados com as personalidades propostas pelo MBTI.

Está em andamento também a evolução do Framework FGT que suporte a identificação da personalidade dos alunos e apoie a sua alocação nos grupos de atividades da disciplina, seja ela presencial ou virtual. Para isso, uma ferramenta tecnológica de suporte à definição de grupos de trabalho em sala de aula está sendo projetada. Esta ferramenta visa executar o processo Formar Grupos de Trabalho (Figura 4), considerando desde a definição da personalidade do aluno até a sua alocação nos grupos de trabalho para execução da tarefa proposta pelo professor.

\section{Referências}

Ciampone, Maria Helena Trench, \& Peduzzi, Marina. (2000) "Trabalho em equipe e trabalho em grupo no programa de saúde da família", In: Revista Brasileira de Enfermagem, 53(spe),143-147. https://dx.doi.org/10.1590/S003471672000000700024

Cohen, E. G., Lotan, R. A. (2017) "Planejando o Trabalho Em Grupo - Estratégias Para

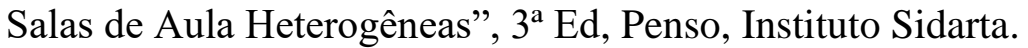

Farias, A., Gomes, T., Cabral, G. (2014). Uma proposta metodológica para o ensino de programação baseado na relação entre perfis cognitivos, padrões pedagógicos e auto regulação dos estudantes. XXXIV Congresso da Sociedade Brasileira de Computação, XXII Workshop sobre Educação em Computação.

Felder, R. M. and Brent, R. (2005) "Understanding student differences". Journal of Engineering Education, 94(1):57-72.

Gessinger, R.M. (2008) “Atividade em grupo, In: A Gestão da Aula Universitária na PUCRS", Editado por Grillo, M.C., Freitas, A.L.S., Gessinger, R.M., Lima, V.M.R., EdiPUCRS, Porto Alegre.

Myers, I. B., Myers, P.B. (1995) "Gifts Differing: Understanding Personality Type" Mountain View, CA: Davies-Black Publishing, 1995.

Nichols, J. D., Miller, R. B. (1994) “Cooperative Learning and Student Motivation”, Contemporary Educational Psychology, 19(2), 167178.doi:10.1006/ceps.1994.1015

Oliveira, E., Sales, G., Pereira, P., \& Moreira, R. (2018). Identificação Automática de Estilos de Aprendizagem: Uma Revisão Sistemática da Literatura. In $26^{\circ}$ Workshop sobre Educação em Computação (WEI 2018). Porto Alegre: SBC. 
VIII Congresso Brasileiro de Informática na Educação (CBIE 2019)

Anais do XXX Simpósio Brasileiro de Informática na Educação (SBIE 2019)

Ribeiro, A. C. R., Longaray, A. N. C., Behar, P. A. (2013) "Estratégias Pedagógicas para a Educação a Distância: um olhar a partir dos estados de ânimo do aluno", In: Congreso Internacional de Informática Educativa, 2013, Porto Alegre. Nuevas ideas en Informática Educativa - TISE. Santiago de Chile: Universidad de Chile. v. 9. p. 606-610.

Silva, V., Castro, R., Duboc, A. L., Motta, C. (2017) "Mineração de Dados Utilizando Análise de Redes Social para Identificar Tendências de Participação em Aulas Presenciais", In: Brazilian Symposium on Computers in Education (Simpósio Brasileiro de Informática na Educação-SBIE) (Vol. 28, No. 1, p. 1467).

Toseland, R.W., Rivas, R. F. (2005) “An introduction to group work practice”, Boston: Allyn \& Bacon. 64-91.

Van Knippenberg, D., Schippers, M. C. 2007. Work group diversity. Annual Review of Psychology, 58: 515-541. 